\title{
LA AUTONOMÍA DE LA MUJER EN RELACIÓN CON EL TRABAJO REMUNERADO
}

\author{
WOMAN'S AUTONOMY IN RELATION TO PAID WORK
}

José María Uris Lloret

Doctor en Derecho - España

joseull@outlook.es

Resumen Entre 1960 y 1980 las mujeres se fueron incorporando al trabajo remunerado de forma más numerosa, de hecho, las tasas oficiales de actividad femenina pasaron del $20,1 \%$ al inicio de la década de los sesenta al 32,6\% en el año 1979, originando un cambio transcendental tanto en el entorno laboral como en el ámbito social. Son múltiples los estudios que se han realizado y se pueden realizar al respecto, el que les presentamos tiene la finalidad de analizar en qué medida la participación de la mujer en el mercado de trabajo ha facilitado su autonomía personal y cómo ha contribuido a que se avanzara en la igualdad de género. El desarrollo del artículo se ha centrado en las circunstancias en que se desenvolvieron las trabajadoras que accedieron a los centros productivos en la década de los sesenta y setenta del siglo anterior, para posteriormente analizar en qué situación se encuentran las mujeres actualmente en el mercado laboral, la discriminación de la que son objeto, a qué limitaciones están sujetas y qué perspectivas les puede deparar la era digital. En fin, cómo pueden afectar todos esos hechos a su carrera profesional y, en consecuencia, a su autonomía personal.

Palabras clave: mujer, autonomía, trabajo, igualdad, género.

Abstract Between 1960 and 1980, women began to take up paid work in greater numbers. In fact, official rates of female activity rose from $20.1 \%$ at the beginning of the 1960 s to $32.6 \%$ in 1979 , originating a transcendental change in both the working environment and the social sphere. There are many studies that have been carried out and can be carried out in this issue. The aim of the one we present here is to analyse how women's participation in the labour market has facilitated their personal autonomy and how it has contributed to advancing on gender equality. The development of the present article has focused on the circumstances in which women who entered the productive centres in the sixties and seventies of the previous century found themselves, in order to analyse the current situation of women in the labour market, the discrimination to which they are subjected, the limitations they are subject to and what perspectives the digital era may hold for them. In conclusion, how all these facts may affect their professional careers and, consequently, their personal autonomy.

Keywords: woman, autonomy, work, equality, gender. 


\section{Introducción}

El trabajo que les estamos presentando tiene la finalidad de analizar en qué medida la participación de la mujer en el mercado laboral ha facilitado su autonomía personal $y$, en consecuencia, ha posibilitado que se lograran importantes avances en la igualdad de género.

Por lo expuesto, se puede observar que pretendemos examinar tres espacios muy complejos, por lo que tienen de inherentes al desarrollo de la persona, así como al equilibrio de la relación entre las mujeres y los hombres, además, centrándonos en un ámbito como el laboral que entraña una profunda dificultad por sus connotaciones sociales, económicas y personales. Más, si cabe, al focalizarlo en el mercado de trabajo de nuestro país, que por las circunstancias singulares que le acontecen, como analizaremos más adelante, es sumamente difícil poder desenvolverse en él. Por lo tanto, nuestro análisis, debido a las limitaciones que tiene un artículo de estas características, solo abordará algunas cuestiones, las que a nuestro juicio consideramos que puedan ser de más relieve en el momento actual.

A nuestro modo de ver, los tres campos que estamos examinando, autonomía, igualdad de género y mundo del trabajo, tienen una extensión indefinida, sobre todo los dos últimos citados. Porque la investigación sobre dichos espacios es tan amplia que no tiene unos límites concretos y continuamente se están publicando artículos al respecto de sumo interés, dado lo importante que es para las personas tener un determinado grado de autonomía, un trabajo que les permita disfrutar de un cierto bienestar, tanto material como personal, y el poder realizar sus proyectos en igualdad de oportunidades que los otros miembros de la comunidad.

Expuestas las anteriores consideraciones, nos vamos a detener brevemente en las tres áreas señaladas como paso previo al desarrollo del trabajo, donde se analizará la relación que existe entre las mismas y en qué medida dicho enlace puede favorecer o limitar que se avance en la igualdad de género.

Los tres conceptos a los que nos hemos referido son fundamentales en el progreso de las personas, por lo tanto, a nuestro juicio, es indiferente el orden en que los abordemos. En primer lugar, vamos a tratar la autonomía personal, porque todas las personas aspiramos a tener mayor grado de autosuficiencia, debido a que lo asociamos, entre otras cuestiones, a tener más independencia y mayores espacios de libertad. A este respecto, Álvarez (2015) señala:

La autonomía no se dirime solo en primera persona, que no es solo un indicador de las habilidades cognitivas del sujeto racional, sino que el desarrollo de dichas capacidades está fuertemente condicionado por elementos externos al sujeto, por el contexto y por las relaciones que en el marco de dicho contexto el sujeto entabla. (p.20) 
En segundo lugar, analizaremos la igualdad de género. Como afirma Fernández (2003), "la igualdad es una de las ideas morales más intuitivas. Cualquier actuación, comportamiento o situación que sean contrarios a las exigencias de la igualdad suelen suscitar una inmediata desaprobación, un inmediato rechazo por considerarlos injustos" (p.15). Por ello, amplios sectores de la ciudadanía consideran que es necesaria la igualdad de derechos y oportunidades entre mujeres y hombres en todos los ámbitos. Aunque lo cierto es que ese camino no está siendo fácil de recorrer, más bien está lleno de obstáculos que resultan incomprensibles, ya que es ineludible la igualdad entre las personas en todos los espacios, además de ser un hecho indispensable para que exista justicia social y podamos lograr un estado de bienestar. A partir de ese logro, en nuestra opinión, se podrá avanzar en la erradicación de otros tipos de discriminación que tanto lastran el progreso de la humanidad.

Cada vez es más evidente que el mayor error que se ha cometido en la historia de la humanidad ha sido la discriminación a la que han sido sometidas las personas del sexo femenino. Sustraer al conjunto de la población de lo que podían haber aportado esas mujeres durante siglos es una lacra que nunca se podrá superar, es inmenso el capital intelectual que no se ha aprovechado y el tiempo que se ha perdido, un abismo sin fin del que nunca sabremos su profundidad, además de la frustración que han sufrido esas personas ante las limitaciones que se les imponían y se les siguen imponiendo. Por estos hechos, resulta muy difícil de entender la larga agonía del patriarcado, con las consecuencias tan graves que tiene en todos los campos.

En tercero y último lugar, vamos a examinar lo que representa el trabajo en nuestras vidas. Además de proporcionarnos una remuneración que puede facilitarnos cierto bienestar, si realizamos una labor que consideramos que es de utilidad para el conjunto de la sociedad, nos produce satisfacción, da sentido a nuestra existencia, repercutiendo favorablemente en la salud y en la autoestima. Por el contrario, si para poder obtener unos ingresos realizamos un trabajo que no es de nuestro agrado y al que no le encontramos sentido, nos puede originar frustración y tener consecuencias negativas en nuestro estado de salud mental y física. Como afirma Ovejero (2006), "el concepto de trabajo es algo más complejo que la mera actividad para conseguir el sustento" (p.17). Por lo que hemos expuesto, se puede observar el papel central que tiene el trabajo en la vida de las personas trabajadoras y la diversidad de elementos que contiene.

En este sentido, Rojas Marcos (2010) afirma:

Para la mayoría de las personas el empleo significa la fuente de seguridad material, el medio de conseguir el sustento diario de ellas y de sus familias. Es más, para muchos constituye una fuente positiva de identidad personal y social que les ayuda a definirse, a valorarse y nutre en ellos el sentido de la propia competencia y la satisfacción con la vida. (pp.24-25) 
Para finalizar este epígrafe, queremos incidir en que la autonomía personal está muy condicionada por las circunstancias en que se desenvuelve la persona, tanto por sus características físicas y psíquicas como por el entorno con el que se relaciona. "En otras palabras, la condición de independencia debe plantearse incluyendo, en primer lugar, la capacidad de la persona para reflexionar sobre sí misma, el entorno y las otras personas" (Álvarez, 2015, p.17).

Por lo que consideramos que es importante desarrollar en el estudio que les estamos exponiendo cómo ha evolucionado la situación de la mujer en el ámbito laboral, las perspectivas que tiene en un futuro próximo, la discriminación que ha sufrido y sufre, a qué limitaciones está sujeta o ha estado en un tiempo reciente, condicionando, por tanto, su labor y afectando negativamente su carrera profesional.

\section{Mujer y trabajo remunerado}

Para examinar en qué medida la participación de las mujeres en el trabajo remunerado ha sido positivo para que se avanzara en la igualdad entre ambos sexos, siguiendo a Carrosca Bengoa (2008) consideramos que para "analizar el trabajo de las mujeres exige antes que nada una reflexión sobre el propio concepto de trabajo" (p.146).

Por lo que debemos observar el valor que se le asigna a dicho concepto en la actualidad, ya que en el transcurso del siglo pasado ha evolucionado el sentido de esa actividad y, además de su significado económico, se valora cada vez más lo que representa para nuestro desarrollo como personas.

El trabajo ocupa un espacio importante en nuestras vidas: le dedicamos más de un tercio de las veinticuatro horas de los días laborables, si tenemos en cuenta que nos recomiendan que debemos dormir ocho horas diarias, tenemos como resultado que, en teoría, más de la mitad del tiempo que estamos despiertos los empleamos trabajando. Como señalan Luceño, Martín, Rubio y Jaén (2008) "la mayoría de nosotros pasamos más horas al cabo de la semana en nuestros respectivos lugares de trabajo que en nuestros hogares o fuera de ellos, disfrutando del tiempo libre con la familia o amigos" (p.112).

Decíamos en el párrafo anterior que, en teoría, una parte significativa del día lo empleamos trabajando, porque en la práctica es mucho más. Dado en el contexto en que estamos viviendo, debemos estar reciclándonos y a veces hasta reinventándonos con frecuencia, porque en la actualidad al contrario de lo que sucedía unas décadas atrás, y sobre todo en el mundo laboral, parece que todo es provisional y lo que es válido ahora, dentro de poco parece que ya es obsoleto. En este sentido, Fernández Collados (2020) afirma que "la formación profesional continua es esencial en una industria tan cambiante" (p.128). 
También debemos observar que en nuestra vida laboral se nos originan una serie de problemas legales y/o daños a la salud u otros asuntos, que tenemos que destinar una parte de nuestro tiempo libre a consultar a profesionales expertos en temas laborales, de la salud, etc., de lo que podemos deducir que, en nuestra vida cotidiana, las tareas productivas tienen un significado muy trascendental.

Si a lo descrito en los párrafos anteriores le añadimos que una parte importante de nuestra existencia la dedicamos a aprender un oficio, podemos inferir claramente el lugar que ocupa nuestra carrera profesional en nuestras ilusiones y en nuestras metas, no solo representa unos ingresos económicos, supone también nuestros proyectos y es uno de los pilares más sólidos de nuestra autoestima.

Asimismo, el trabajo es la base de la actividad económica de las sociedades desarrolladas, sin la producción de bienes y servicios no es posible el funcionamiento de la sociedad contemporánea y el más grave problema social que puede tener un estado es que un número importante de sus habitantes no tengan acceso a un empleo, como tenemos constancia en nuestro país desde hace décadas.

Además de la importancia que tiene para las personas tener un trabajo remunerado, así como para el estado que su población pueda acceder a un empleo, tenemos que una parte de nuestro tiempo de ocio con frecuencia lo dedicamos a realizar una tarea, bien colaboramos con alguna ONG, hacemos bricolaje, cocinamos con ilusión alguna receta, arreglamos el jardín, moldeamos una pieza de cerámica, etc., en fin, una parte sustancial de nuestro quehacer está asociado con alguna labor, como afirman Castillo y Agulló (2012) "el trabajo invade todas las esferas de la vida" (p.17).

Conocedor de lo importante que es el trabajo para el desarrollo de los pueblos, la cultura masculinista ha controlado totalmente esta faceta de las personas, reservando el trabajo visible y mejor remunerado para los varones y el trabajo invisible sin remunerar o escasamente remunerado para las mujeres porque, como señala Millett (2010), "el patriarcado es una ideología dominante que no admite rival; tal vez ningún otro sistema haya ejercido un control tan completo sobre sus súbditos" (p.82).

Pese a esa discriminación que ha realizado el orden androcéntrico en el ámbito laboral, en mayor medida que en otros espacios, por considerarlo un campo exclusivo de los varones, "la mujer ha entendido que su liberación pasaba por trabajar fuera de casa y equipararse al varón en cobrar por su trabajo" (Camps, 1998, p.10).

En este sentido, Sarasúa y Gálvez (2003) exponen que:

Es precisamente el acceso masivo de las mujeres al empleo asalariado, y sobre todo a la educación y a las profesiones mejor consideradas y remuneradas, lo que ha hecho que la desigualdad sea un problema económico y político, y lo que ha acabado transformando la situación jurídica, política y económica de las mujeres. (p.32) 
Porque como bien señalan Martínez y Paterna (2009): "La invasión del empleo en nuestras vidas guarda una intensa relación con las necesidades de identidad, prestigio y reconocimiento social" (p.26). Por lo tanto, cuando se discrimina a las mujeres en el ámbito laboral, se las perjudica económicamente y también su realización como persona, así como su carrera profesional.

Debido al papel central que tiene en la actualidad el trabajo en la vida de las personas, como hemos referido en las líneas anteriores, estamos en la sociedad de la ciudadanía laboral, pero, como afirma Alonso (2007), es "una ciudadanía laboral definida como masculina" (p.28). Porque

el Derecho del Trabajo en Europa se creó pensando en un trabajador hombre, del sector industrial, a jornada completa y responsable del sostén económico familiar, que no precisa medidas de conciliación; este modelo define los derechos laborales y la organización del trabajo. (Conde-Pumpido, 2007, pp.20-21)

Como se ha expuesto en los párrafos anteriores, las mujeres se han ido incorporando al mercado de trabajo de forma masiva en las últimas décadas, como la opción más lógica al llegar a la edad adulta para poder realizar sus proyectos profesionales, pero los centros productivos no han modificado su organización, que sigue definida en la idea de que la persona que está realizando la labor es un varón sin obligaciones familiares, porque las realiza su esposa.

De esta forma las personas trabajadoras están sometidas a unos horarios que no les facilitan atender sus necesidades personales. Asimismo, en los centros de trabajo no tienen, en la mayoría de los casos, los servicios adecuados para atender a sus hijos, en fin, existe un déficit muy significativo en materia de conciliación familiar que perjudica claramente la carrera profesional de las mujeres, así como su salud, porque sigue vigente una mentalidad patriarcal que asigna las labores de cuidado a ellas.

\section{En qué circunstancias se incorporaron las mujeres al trabajo remunerado de forma más numerosa}

Consideramos que para realizar un análisis en profundidad de la situación de las trabajadoras en el mercado de trabajo actualmente y de las cuestiones que les afectan, es necesario observar a qué circunstancias se enfrentaron al acceder a los lugares de trabajo de forma más numerosa entre 1960 y 1980.

En primer lugar, porque valoramos que es importante que no queden en el olvido las situaciones tan adversas que tuvieron que afrontar esas mujeres para realizar un acto tan noble y fundamental en la vida de las personas como es el trabajo; en segundo lugar, porque, a pesar de los años que han transcurrido, muchos de los problemas a los que tuvieron que hacer frente, aún siguen vigentes e impiden que se avance en la igualdad de género. 
Pero es importante resaltar que las dos décadas a las que hemos hecho referencia al iniciar el epígrafe fueron trascendentales en la presencia de las mujeres en los lugares de trabajo en España. De hecho, en ese momento histórico, se sentaron una parte importante de las bases que facilitarían el desarrollo de la carrera profesional de las mujeres, logrando un mayor grado de autonomía y avanzando en igualdad de derechos hacia sus compañeros varones.

Aunque es importante recordar que no fue un tiempo fácil, más bien todo lo contrario, por lo que entendemos que el análisis que pretendemos realizar sobre algunas cuestiones que afectan a la igualdad de género en el ámbito laboral no se transmitiría de forma íntegra si no nos detenemos en dicha coyuntura.

Si a las mujeres de los países occidentales les resultó muy difícil acceder a un espacio que el patriarcado solo tenía reservado para los hombres y ellas se tenían que conformar con ciertos empleos muy feminizados o con los puestos de trabajo que para ellos no tenían interés, en España, dadas las circunstancias históricas que acontecían en ese momento que describiremos en los párrafos siguientes, las españolas tenían enfrente un muro que, a diferencia del de Berlín, era mucho más difícil de superar y aún no ha sido derribado del todo.

Porque cuando las mujeres en los años sesenta del siglo veinte incrementan su presencia en los centros de trabajo de nuestro país, lo hacen bajo el gobierno de una dictadura que se prolongaba más de veinte años y las relaciones laborales tenían el germen de su regulación en el Fuero del Trabajo que "asignó a la figura paterna el estatus de "jefe del hogar», dotado de privilegios familiares y materiales como la cualificación y el salario, y trató de poner barreras de acceso al trabajo remunerado de la mujer casada" (Vilar, 2012, p.119).

Expuestas las anteriores consideraciones, nos situamos en los hechos a que hacemos referencia. Las mujeres que habían nacido en la posguerra y en los años siguientes, fueron las primeras generaciones del sexo femenino que, al llegar a la edad adulta, en la década de los sesenta y setenta, se fueron integrando en una mayor proporción que las generaciones anteriores al ámbito laboral, las tasas oficiales de actividad femenina pasaron del $20,1 \%$ al inicio de la década de los sesenta al 32,6\% en el año 1979 .

Además, accedieron al mercado de trabajo con la voluntad de quedarse, porque lo usual en sus predecesoras era abandonar el empleo al casarse o al quedarse embarazadas, en muchos casos porque así lo disponían las normas laborales vigentes en ese momento. Igualmente, también contribuían en esa decisión los usos sociales de la época que, debido a la ideología del régimen franquista, la mujer casada solo podía ejercer el rol de madre y esposa.

Como señala Jiménez (2009), "hasta los primeros años ochenta del pasado siglo XX, muchas mujeres españolas, al casarse, dejaban un trabajo remunerado para pasar a 
engrosar el enorme elenco de pluriempleadas que los varones clasificaba con un humorístico «ha dejado de trabajar" (p.22).

Aunque un porcentaje importante de trabajadoras en los años sesenta y setenta permanecieron en sus puestos de trabajo, como hemos indicado anteriormente, un número elevado de ellas, cada vez menos, siguieron dejando su puesto de trabajo o pidiendo una excedencia al casarse o tener hijos hasta la década de los ochenta del siglo pasado. A partir de esas fechas, lo usual es que permanezcan en su puesto de trabajo, aunque hayan contraído matrimonio.

Ese cambio de actitud de las mujeres hacia el trabajo remunerado se debió, entre otras cuestiones, a la promulgación de la Ley 56/1961, de 22 de julio, sobre derechos políticos profesionales y de trabajo de la mujer ${ }^{1}$. Su aprobación, según Valiente (1998), "revistió considerable importancia porque amplió el rango de los derechos laborales reconocidos a las mujeres, inició una pauta de desmantelamiento progresivo (pero no total) de las discriminaciones legales referidas al trabajo extradoméstico femenino" (p.50).

También ayudó a este nuevo contexto laboral, en donde las mujeres paulatinamente se estaban integrando, las importantes transformaciones sociales y productivas que se generaron a partir de los años sesenta. En un relativo corto espacio de tiempo se produce una gran transformación en la distribución porcentual de la población activa entre los distintos sectores de la economía nacional, pasando en apenas quince años de estar trabajando la mayor parte de la población en el sector primario a trabajar en el sector terciario, que progresivamente ha ido incrementado su dimensión $y$, en los últimos años, emplea a más de las tres cuartas partes de la población activa.

En lo referente al entorno social, debido al cambio tan notable en el sistema productivo que exponíamos en el párrafo anterior, en el espacio de muy pocos años se produce una migración de una parte importante de la población de zonas rurales a zonas urbanas. Como señala Sergio del Molino (2018) "entre 1950 y 1970 se produjo el éxodo. En menos de veinte años, las ciudades duplicaron y triplicaron su tamaño. Las capitales se colapsaron. En muy poco tiempo, el campo quedó abandonado" (p.28).

Ese cambio tan radical en la vida cotidiana de la gente, así como el desarrollo del turismo extranjero, sobre todo de los países europeos donde la población trabajadora tenía un mayor poder adquisitivo que los españoles y vivía en regímenes democráticos, procedió a traer tímidamente aires de modernidad a la sociedad española, quedando poco a poco atrás los largos años de la postguerra, con sus secuelas de hambre, represión y ausencia de libertad.

${ }^{1}$ BOE núm. 175, de 24 de julio de 1961.

IQUAL. REVISTA DE GÉNERO E IGUALDAD, 2022, 5, 145-167

ISSN. 2603-851X

DOI. http://dx.doi.org/10.6018/iqual.489651 
Pese a esos leves avances, a los que hacemos referencia en el párrafo anterior, cuando las mujeres se iban incorporando de forma más regular a los centros productivos, percibían que las recibían como extrañas, como personas ajenas a ese mundo, en el mejor de los casos; porque también, con frecuencia, advertían que sus compañeros las consideraban una amenaza a su estatus, rechazando explícitamente su presencia en los lugares de trabajo.

Igualmente, tenían que soportar actitudes paternalistas, como si se tratasen de seres inferiores necesitadas de protección, que solo podían ofrecerles los hombres porque se consideraban superiores. Como bien afirman Rubio y Gil (2012, p. 133) "la presencia femenina en el entorno de trabajo es percibida con desagrado y hostilidad en un contexto de dominación tradicional masculina, donde además la mujer se erige como potencial competidora".

Las conductas descritas y otras que no hemos traído a colación, pero que son conocidas por la manera tan injusta que se trató a las personas del sexo femenino al acceder a un empleo, estaban originadas por siglos de discriminación de las mujeres y por haber considerado que el trabajo remunerado era un espacio privado de los hombres.

En nuestro país, esas acciones se acentuaron debido al modelo arcaico que impuso la ideología franquista, que expulsaba a las mujeres del ámbito público permitiéndoles solo su realización en el hogar familiar. Asimismo, por la prohibición de la educación mixta, obligando a todos a una enseñanza confesional, católica y que se separaba por sexos. Esa tesitura, según Martín Gaite (2017, p. 96).

Marcó sensiblemente la conducta de las nuevas generaciones de españoles en su paso de la infancia a la pubertad; y esto se acusaba en la intrínseca dificultad para la «camaradería» latente en las pocas chicas que llegaban a la universidad o a trabajar en una oficina, porque además tampoco los hombres con que iban a alternar allí se prestaban, salvo honrosas excepciones, a un trato sin reticencias.

Pero no solo fueron las agresiones físicas, psíquicas, verbales y el tratarlas de forma despectiva las únicas situaciones adversas a las que se enfrentaron esas trabajadoras. También tuvieron que hacer frente a la nula prestación de servicios por parte de las Administraciones Públicas, el único apoyo que tenían era el de la familia, en los casos que vivieran en el mismo municipio, porque sus madres generalmente no realizaban un trabajo remunerado y podían cubrir ese espacio, que ni la administración ni las empresas facilitaban.

No obstante, pese a todas esas dificultades, esa generación de mujeres que situamos aproximadamente entre 1965 y 1980, interiorizaron, como no habían hecho las anteriores generaciones, que tenían el mismo derecho que los varones a trabajar en las organizaciones productivas y esa esencia la transmitieron a las generaciones posteriores; que cada día están mejor formadas, teniendo una presencia superior en los 
centros de formación, además con muy buenos expedientes académicos, pese a conocer las barreras que tienen que superar en los lugares de trabajo, lo que supone un espíritu de superación digno de subrayar.

A este respecto, Julián Marías (1979) expone que "generación histórica es un plazo de aproximadamente quince años" (p.127) porque en ese período de tiempo "se produce un cambio social, un cambio en las estructuras del mundo, un cambio global que afecta a todas las dimensiones de la vida" (Marías, 1979, p.127).

Nosotros, como hemos indicado en las líneas anteriores, entendemos que desde finales de la década de los sesenta a los primeros años del decenio de los ochenta se produce un cambio transcendental del papel de la mujer, respecto al trabajo remunerado, con relación a generaciones anteriores, que daban más prioridad al matrimonio. Martín Gaite (2017) en su ensayo Usos amorosos de la postguerra española nos deja constancia de esa situación. "De vez en cuando, en las publicaciones de la época, se hacen encuestas a las chicas que estudian o que trabajan y casi todas contestan lo mismo: que cuando se casen dejarán de hacerlo" (...) "Incluso cuando habían llegado a una carrera de categoría, la tomaban como algo provisional. Su verdadero ideal era otro. O al menos eso es lo que decían de forma casi unánime" (p.50).

Tal y como subraya Kierkegaard (1972, p. 11) "cada generación tiene una misión". Y esa generación de mujeres que en su juventud vivieron el final de la dictadura y el inicio de la democracia, influidas seguramente por ese nuevo tiempo, el acceso en mayor número a la enseñanza superior y observando el papel secundario al que se había relegado a su colectivo a lo largo de la historia de la humanidad, entre otras cuestiones, valoraron, como no se había hecho anteriormente, la independencia financiera que ofrecía un empleo.

Esas circunstancias modificaron notoriamente la situación de la mujer en la sociedad, era necesario otro escenario, no se podía demorar más la discriminación a la que era sometida. La prolongación de dicha marginación solo depararía consecuencias negativas para el conjunto de la ciudadanía y, debido a los importantes avances que se han realizado, esperemos que dentro de pocos años no leamos en la prensa titulares sobre la brecha salarial, el techo de cristal, el bajo número de mujeres directivas, las escasas facilidades para la conciliación de la vida familiar, etc.

\section{La situación de la mujer en el mercado de trabajo en la actualidad}

El desarrollo del artículo que les estamos presentando ha coincidido en el tiempo con la publicación del barómetro del Centro de Investigaciones Sociológicas, correspondiente al mes de junio de 2021, en el que el paro, según las personas encuestadas, vuelve a ser el principal problema de la población española, teniendo una eco importante dicha noticia en los medios de comunicación.

IQUAL. REVISTA DE GÉNERO E IGUALDAD, 2022, 5, 145-167

ISSN. 2603-851X

DOI. http://dx.doi.org/10.6018/iqual.489651 
Es de destacar, como subrayan los medios, que el desempleo sea el asunto que más preocupa a los habitantes de nuestro país, cuando estamos inmersos en una pandemia que no sabemos cuándo finalizará, ni las consecuencias que puede tener en la salud y la economía; pero es que desde hace varias décadas el mercado de trabajo en España tiene un alto paro estructural y se ha convertido en un escenario muy difícil para que las trabajadoras y trabajadores puedan llevar a término sus aspiraciones profesionales.

Porque, desde aproximadamente 1975 , buscar empleo o mantenerlo es un hecho sumamente complejo en nuestro país. Las cifras de desempleo desde esa época han sido siempre elevadas, a excepción de los primeros años del siglo XXI, pero con muchas matizaciones, porque el bienestar del que tanto se presumía al inicio del milenio estaba un tanto maquillado.

En el ecuador de la década de los setenta del siglo anterior concurrieron una serie de circunstancias negativas que supusieron una coyuntura muy crítica para la economía, que afectó muy desfavorablemente al empleo, teniendo una clara repercusión en las condiciones de trabajo y en los salarios.

En el plano internacional, la crisis del petróleo de 1973 supuso la quiebra del sistema, como afirma Oñate (2010) al exponer que:

El incremento del déficit público y la deuda pública -como medios para tratar de reactivar el sistema- y las carencias del Estado para gestionar con la suficiente eficacia todos los asuntos de unos sistemas políticos extendidos y expandidos y cada vez más complejos acabaron conduciendo a la crisis del Estado de bienestar.

(pp.202-203)

$\mathrm{Si}$, a nivel global, la crisis que se originó a raíz del fuerte incremento del precio del crudo generó importantes tensiones, en el ámbito laboral tuvo una incidencia notable debido a las elevadas tasas de inflación y la pérdida de poder adquisitivo de los salarios, ya que "para hacer frente a esta situación se aplicaron duras medidas, que ponían en peligro las conquistas salariales y sociales de las décadas anteriores" (Vilar, 2012, p.116).

En nuestro país, a esas difíciles circunstancias hay que adicionarle la incertidumbre política que se vivía en esos años debido a coincidir en el tiempo con la finalización de la dictadura y el período de transición a la democracia, que demoró las medidas que se debían de adaptar para hacer frente a esa crisis mundial.

De lo que se puede deducir que las personas que iniciaban su camino en el ámbito laboral, en el que se encontraban las jóvenes y no tan jóvenes que consideraban que debían realizar una carrera profesional, no lo hacían en el marco más idóneo sino todo lo contrario, en uno sumamente adverso, al que había que sumarle los trabajadores que habían emigrado a otros países que ahora retornaban debido a la crisis energética, por lo que las cifras de desempleados, que ya eran muy altas, se incrementaron significativamente en un país que durante la dictadura se había mantenido en la ficción del pleno empleo. Invención que había perdurado en el tiempo por la migración de un 
número significativo de personas por causas económicas hacia otras naciones y la discriminación de las mujeres en el ámbito laboral.

Las circunstancias descritas en los párrafos anteriores, entre otras, así como la perduración de una mentalidad patriarcal que no contemplaba la concurrencia de las mujeres en los centros productivos con los mismos derechos que los hombres, más en una situación de alto desempleo en el que entendían que se debía priorizar a los varones por estar presente aún el modelo tradicional de que era el cabeza de familia y tenían que traer un jornal al hogar. Esta situación no predecía un futuro muy favorable para las trabajadoras, como los hechos han demostrado ya que, con frecuencia desempeñan actividades temporales encuadradas en grupos profesionales de escasa calificación, remuneración y estatus social (Millán, Santos y Pérez; 2015).

Esa situación tan complicada que han tenido y tienen las relaciones laborales en España han originado que el Estatuto de los Trabajadores haya sido modificado en varias ocasiones, así como que se hayan realizado un número importante de reformas laborales. Afirmando Vilar (2012), a este respecto, "la impresión de vivir en un Estado de permanente reforma del mercado laboral" (p.118).

Con esos desequilibrios en el mercado laboral, la crisis financiera del 2007 que tuvo una importante incidencia sobre el empleo a nivel global en nuestro país tuvo un efecto devastador. Tanto que a principios del año 2009 las cifras de desempleo parecían imparables suscitando una gran preocupación social ante el futuro más próximo, paralizando en una medida importante el consumo de las familias, dando lugar a un círculo cerrado (o vicioso) en el que el miedo retraía el consumo de los ciudadanos, lo que provocaba una ralentización en la economía, originando más paro.

Esas circunstancias originaron que la precariedad, que ya estaba presente en el ámbito laboral desde hacía unas décadas, se hiciera mucho más intensa y su dimensión más importante, haciéndose más patente la figura del trabajador pobre, que como afirma Auvernong (2012) "no corresponde propiamente a un concepto jurídico, más bien se refiere a un fenómeno sociológico caracterizado por personas que a pesar de trabajar y recibir una remuneración, tienen un ingreso familiar por debajo del umbral de pobreza". En este sentido, San Martín (2020) señala:

Es precario el trabajo que viene condicionado por la ausencia de oportunidades de trabajo aceptables, de remuneración adecuada, de jornada laboral limitada, de estabilidad y de seguridad en el empleo, de trato justo en la contratación y condiciones laborales, de protección social, de posibilidad de compatibilizarlo con la vida familiar y personal. (p.266)

En base a lo anterior, se puede deducir las dificultades que tienen las personas trabajadoras para conseguir sus fines. Y si en líneas generales la situación es complicada para ciertos colectivos como mujeres, jóvenes, inmigrantes, etc., el escenario es 
sumamente difícil, más si cabe, si eres una joven que has migrado de otro país para trabajar en España.

A este respecto, Carrasquer (2007) expone que "la desregulación de la jornada laboral puede leerse también en clave de mayor precariedad femenina y de refuerzo de las desigualdades de género. Unas desigualdades que acrecientan su presencia en el mercado laboral junto a las de etnia" (pp.147-148).

Los hechos expuestos inciden en que un número significativo de mujeres tengan serias dificultades para hacer frente a sus gastos financieros, coyuntura que se agrava si tienen hijos a su cargo. Según la Encuesta Continua de Hogares (INE, 2020, p. 5) el $81,4 \%$ del total de hogares monoparentales estaban integrados por una madre con sus hijas e hijos. Asimismo, según la Encuesta de Condiciones de Vida del año 2019 (INE, 2019, p. 7) el 41,4\% de las familias monoparentales está en riesgo de pobreza.

Como indica el Análisis de las necesidades sociales de mujeres y hombres, publicado por el Observatorio Social de la Caixa en junio de 2020:

La tasa de riesgo de pobreza monetaria, privación material o pobreza consistente de las personas que viven en hogares monoparentales encabezados por una mujer duplican las tasas correspondientes a la población en su conjunto y, en todo caso, son siempre mucho mayores que las de los hogares monoparentales encabezados por hombres. (Ayala Cañón; Cantó Sánchez; Martínez López; Navarro Ruíz y Romaguera de la Cruz; 2020, p.11)

En este sentido, el estudio realizado por la Federación de Asociaciones de Madres Solteras (S.F.) sobre las Familias Monoparentales en España, señala que: "En este modelo familiar se materializa la feminización de la pobreza, que se pone de manifiesto en el empobrecimiento diferencial de los hogares monoparentales encabezados por mujeres. La desigualdad de género atraviesa a estas familias en las esferas laboral, familiar y política" (p.127). Los datos expuestos nos llevan a citar las palabras de Millet (2010): "el que una mujer sea cabeza de familia se considera una eventualidad poco deseable, señal únicamente de pobreza o de alguna desgracia" (pp.83-84).

Los datos que hemos expuesto en los párrafos anteriores sobre los hogares monoparentales nos indica las dificultades que tienen las familias que se encuentran en esas circunstancias, pero no nos informa del número de mujeres, que consideramos que debe ser elevado dado los índices de precariedad tan altos que nos muestran las estadísticas, que quieren formar una familia monomarental o que desean finalizar su relación de pareja y lo tienen que aplazar por motivos financieros, en consecuencia ven limitados sus espacios de libertad.

A este respecto, Castells (2007) afirma que "las separaciones del núcleo familiar tienen un coste económico tan alto que el cálculo financiero es uno de los más importantes mecanismos de preservación de la convivencia, a pesar de los conflictos: la familia que se hipoteca unida permanece unida" (p.26). 
Pero es que además esa limitación de su autonomía puede pasar a sus hijas e hijos, porque esas desigualdades pueden persistir de una generación a otra según el proyecto Atlas de Oportunidades que ha realizado la Fundación Felipe González y Cotec (2020):

El hogar en el que un niño crece influye veinte años después en su renta. Los niños de hogares con menores ingresos tienen más posibilidades de permanecer en esa misma posición y los hijos de familias con ingresos más altos tienen más opciones para obtener después una renta superior a la mayoría.

Asimismo, en el estudio "España 2050: fundamentos y propuestas para una Estrategia Nacional de Largo Plazo" que presentó el Gobierno de la nación en mayo de 2021, se indica que "nacer en familias con bajos ingresos en nuestro país se relaciona con peores resultados en renta y desarrollo profesional y reduce significativamente las posibilidades de gozar de una buena salud" (p.329). En dicho estudio se hace referencia a que "en ciudades como Madrid y Barcelona la esperanza de vida media en los barrios más ricos es entre 7 y 11 años más alta que en los barrios más desfavorecidos" (p.329). Igualmente, como afirman Viguer y Serra (1996):

Un aspecto importante que repercute en el desarrollo de los niños es el desempleo de los padres. Cuando el principal mantenedor de la familia -tanto si es el padre como la madre- se queda en paro, la vida familiar cambia de modo predecible, afectando a menudo el curso del desarrollo. Aunque el desempleo afecta a todas las capas sociales, los efectos en la clase obrera son más graves. La mayoría de los efectos del paro en la infancia son indirectos siendo el resultado del cambio de comportamiento y disposición por parte del progenitor que se ha quedado sin trabajo. (p.198)

En base a lo expuesto en los párrafos anteriores, podemos afirmar que la marginación que sufren las mujeres en el ámbito laboral, además de limitar su desarrollo personal y profesional, también condiciona la vida de sus hijas e hijos, con lo que estamos ante una discriminación continua que afecta en primer lugar a la madre, pero que también tiene efectos sobre su familia.

Finalmente, también debemos observar cómo afecta a la pensión de jubilación de las trabajadoras las circunstancias que han tenido lugar en su carrera profesional, porque los importes monetarios que perciba por ese concepto se calculan en base a lo que ha cotizado durante los años de actividad laboral. Por lo que, si su cotización no ha sido muy elevada, la cuantía de su pensión estará en los tramos más bajos. A este respecto, Ayuso y Chulía (2018) indican que:

La actual situación de desventaja femenina en el ámbito de las pensiones contributivas no es deudora de un sistema de pensiones discriminatorio contra las mujeres, sino fundamentalmente de acusadas diferencias en los comportamientos laborales entre los hombres y las mujeres que hoy forman parte de la población mayor española. (p.3) 
Si, además de esa situación desfavorable en el cobro de las pensiones de las trabajadoras, tenemos en cuenta que las mujeres tienen una esperanza de vida mayor que la de los hombres, tienen que estar más años dependiendo de esos ingresos. Asimismo, que ese tiempo no va a ser fácil, porque "presentan mayor morbilidad, discapacidad y una peor calidad de vida" (Blázquez Rodríguez y Montes Muñoz, 2011, p.5). Podemos concluir que las dificultades que tienen en la actualidad las trabajadoras en el ámbito laboral, con toda probabilidad se incrementen significativamente cuando se jubilen.

\section{Las trabajadoras en la industria 4.0}

El cuadro descrito en los epígrafes anteriores, en algunas cuestiones, es bastante desolador y desde luego no presenta el mejor escenario para las trabajadoras y trabajadores, cuando estamos viviendo en un período de tiempo de transición hacia un nuevo modelo socioeconómico y tecnológico, porque está llegando a su fin el ciclo en que el petróleo y los otros combustibles fósiles fueron la base donde se erigió la infraestructura industrial, originando un espacio histórico de cambio que, entre otras cuestiones, genera desorientación e inquietud entre las personas, por no conocer con exactitud a lo que se enfrentan.

Asimismo, en el espacio relativamente corto de tiempo que ha transcurrido del nuevo siglo, hemos sufrido una crisis financiera de una magnitud parecida a la de 1929, que dado el tiempo que había pasado desde aquella recesión económica, pensábamos que no nos íbamos a volver a enfrentar a una conmoción de esa índole y se demoró mucho en el tiempo en poner los medios más adecuados para hacerle frente, afectando ese hecho, entre otras cuestiones, a que tuviese una gran incidencia sobre el empleo, así como sobre las condiciones de trabajo.

Pero no solo hubo impericia en diseñar los controles para que un hecho de esa naturaleza no sucediera, así como en poner los medios más adecuados para hacerle frente. También faltó capacidad para planificar la salida de ese tiempo tan crítico y, cuando aún no se había superado del todo, dado que algunos aspectos de las relaciones laborales, como el salario, entre otros, todavía seguía condicionado por los tiempos difíciles que se habían vivido, nos vimos inmersos en la actual pandemia. Como se indica en el preámbulo de la Ley $2 / 2021$, de 29 de marzo de medidas urgentes de prevención, contención y coordinación para hacer frente a la crisis sanitaria ocasionada por el COVID-19:

En una crisis sanitaria sin precedentes y de una extraordinaria amplitud y gravedad, tanto por el extraordinario riesgo de contagio y el alto número de ciudadanos afectados, con la consiguiente presión sobre los servicios sanitarios, como por el

2 BOE núm. 76, de 30 de marzo de 2021, páginas 35952 a 35980.

IQUAL. REVISTA DE GÉNERO E IGUALDAD, 2022, 5, 145-167

ISSN. $2603-851 \mathrm{X}$ DOI. http://dx.doi.org/10.6o18/iqual.489651 
elevado coste social y económico derivado de las medidas extraordinarias de contención y distanciamiento adoptadas por los distintos Estados.

En base a lo anterior, se puede deducir nítidamente que las circunstancias sociolaborales en que se inicia esta nueva revolución industrial no son las más idóneas y las dificultades se pueden acrecentar, sobre todo para los colectivos que más difícil lo tenían, como es el caso de las mujeres. Pero, como afirma Marina (2006) "nuestra calidad de vida va a depender de cómo seamos capaces de afrontar las situaciones difíciles" (p.40).

$Y$, conscientes de esa situación, porque siempre han tenido que enfrentarse a escenarios muy arduos, las personas del sexo femenino desde hace varios años han hecho un gran esfuerzo en formación, superando claramente a los varones, y ese capital humano con toda seguridad será valorado en una época productiva en "que las empresas que están digitalizando más, también están creciendo más y requiriendo nuevos tipos de trabajos, por lo que necesitan ampliar su capital humano" (Ruíz, 2020, p.384).

Porque, como afirma Pazos (2009), "tenemos una población femenina altamente formada cuyo capital humano se despilfarra en precariedad, subempleo, contratos a tiempo parcial, inactividad, economía sumergida y desempleo, fenómenos que lastran el funcionamiento del mercado de trabajo y merman la productividad".

Y como señala dicha autora, "para pasar del ladrillo al ordenador, podríamos empezar por no seguir despilfarrando el capital humano existente" (Pazos, 2009). Así lo entiende, también, el autor de estas líneas, si España, como tristemente ha sucedido en varias ocasiones en el transcurso de la historia, no quiere perder otra vez el tren de la modernidad, tendrá que potenciar de forma eficiente sus recursos humanos. En ese apartado tendrá que valorar el esfuerzo tan importante que han realizado en formación las mujeres durante las últimas décadas, porque la instrucción va a ser fundamental en la era digital, donde surgirán nuevos perfiles profesionales a raíz de los importantes avances tecnológicos y las necesidades de la ciudadanía.

A este respecto, el informe del año 2016 del Instituto Superior para el Desarrollo de Internet (ISDI) expone que la "transformación digital no consiste única y exclusivamente en la implementación de tecnología. El objetivo de este proceso es adaptarse de la mejor manera posible al nuevo escenario, caracterizado por nuevas exigencias del mercado y nuevos consumidores, buscando la consecución de ventajas competitivas" (p.3). Según dicho informe:

El sexo no es una de las variables que muestre más diferencias significativas en el ámbito de la digitalización profesional. Las ratios de ambos sexos son muy similares con ligeras diferencias a favor de la mujer cuando se trata de profesionales con más de 20 años de experiencia: un 6,2 entre aquellas con más de 20 años de expertise digital. (ISDI, 2016, p.10) 
En base a lo anterior, se puede observar que las trabajadoras en la industria 4.0 están desarrollando tareas relacionadas con el talento y la pericia, situación totalmente distinta a la que tenían en las anteriores revoluciones industriales, que ocupaban los puestos de menos relieve y peor pagados, como señala Medina-Vicent (2014, p. 158):

Se les pagaba por pieza fabricada y sus salarios eran tan bajos que a duras penas les daba para su propia subsistencia, hecho que implicaba una mayor dependencia con respecto al marido y la imposibilidad de desarrollar una vida cómoda y plena en solitario.

De lo que se deduce que su posición en la sociedad era muy débil porque sus salarios no le proporcionaban autonomía financiera, las que estaban casadas dependían totalmente de su marido y las que estaban solteras, si eran jóvenes, dependían de sus padres y, si eran mayores, pasaban muchas necesidades.

La situación actualmente es muy distinta, aunque está muy lejos de ser el estado adecuado, y consideramos que la nueva era productiva que se inicia debía ser el marco idóneo para lograr la igualdad real entre mujeres y hombres, ya que consolidaría los importantes avances realizados en los últimos años y el punto de partida de las trabajadoras es claramente superior al de las otras revoluciones industriales, debido al alto nivel de formación que tienen, fruto de una capacidad de trabajo y esfuerzo que se percibe en todos los ámbitos de la sociedad.

\section{Conclusiones}

Iniciábamos el trabajo, que está a punto de concluir, con la voluntad de analizar en qué medida la participación de las mujeres en el trabajo remunerado ha incrementado su autonomía personal. En relación a dicho razonamiento, hemos observado que existen distintos criterios sobre si el acceso de la mujer al mercado de trabajo puede facilitar su independencia y, consecuentemente, que se avance en la igualdad de género.

Por una parte, entre otras autoras, Simone de Beauvoir (2019) señala que "gracias al trabajo la mujer conquistó su dignidad de ser humano, pero fue una conquista especialmente dura y lenta" (p.180). Por otra parte, entre otras voces, Federici (2013) expone "que «dejar la casa» e «ir a trabajar» fuera una condición previa para nuestra liberación es algo que, ya en los años setenta, algunas feministas se habían cuestionado" (p.94).

En relación a lo expuesto en los párrafos anteriores y respetando la diversidad de criterios que se puedan haber expresado sobre la cuestión que estamos examinando, nosotros consideramos que el trabajo remunerado es un punto de apoyo fundamental para la independencia financiera de las mujeres, pero como afirma Hirigoyen (2008) "es una condición importante para la autonomía de las mujeres, pero no es suficiente" (p.36). 
No es suficiente porque, aunque es una realidad la presencia de la mujer en el ámbito productivo, aún permanece una mentalidad tradicional que la sitúa en un lugar secundario, además la responsabiliza de las labores del hogar y de cuidado sin facilitar alternativas que permitan que desarrolle su carrera profesional en igualdad de condiciones que sus compañeros varones. "Se han convertido en mujeres orquesta, que asumen (casi) solas los papeles femeninos tradicionales y al mismo tiempo las actividades antaño reservadas a los hombres" (Hirigoyen, 2008, pp.34-35).

Asimismo, en el desarrollo del trabajo se ha mostrado que, aunque las mujeres se han incorporado de forma masiva a los centros productivos, las organizaciones de trabajo están diseñadas con una vocación androcéntrica que limitan considerablemente la carrera profesional de las trabajadoras. Es necesario un cambio trascendental en los modelos organizativos que contemple que a esos centros de trabajo concurren personas de ambos sexos que deben conciliar producción y reproducción, facetas igual de importantes para el progreso de la sociedad.

El mantener en el olvido esos hechos tan evidentes ha originado que las mujeres asuman "un nuevo papel en el mundo laboral sin que se produzca una redistribución del trabajo doméstico, recayendo sobre ellas el mayor peso de estas responsabilidades. Esta situación ha generado lo que se conoce como la «segunda jornada» laboral de muchas mujeres" (Cebrián y Moreno, 2008, p.367).

Debido a los argumentos que hemos expuesto, nosotros, aunque consideramos que la independencia económica que proporciona una nómina tiene un valor fundamental en la autonomía de la persona, entendemos que se debe observar cual es la situación de la mujer, tanto en el mercado de trabajo como en las organizaciones laborales, así como cuál es su papel en la sociedad, porque no solo el hecho de acceder a un empleo va a posibilitar la igualdad de género, la situación es mucho más compleja y aún queda una parte muy importante del camino por recorrer.

Porque, como afirman Sarasúa y Gálvez (2003, p.13): "Mujeres y hombres desempeñan distintas funciones en los hogares, muestran comportamientos diferentes en los mercados de trabajo y son tratados como mano de obra distinta por los empleadores".

Asimismo, como afirman Millán, Santos y Pérez (2014) resulta una condición indispensable la equiparación en todos los niveles (horizontal y vertical) para alcanzar un mercado de trabajo igualitario, pues de no ser así, se correría el riesgo de desaprovechar la mitad de la masa laboral, al relegar al colectivo femenino en favor del masculino.

Por lo que es necesario e inaplazable aplicar en todos los espacios del ámbito laboral la perspectiva de género, las mujeres deben estar al mismo nivel que los hombres en todos los aspectos que conciernen a las relaciones laborales ya que el trabajo femenino no es subsidiario del masculino y no son admisibles los estereotipos, roles y modelos 
productivos que discriminan a las trabajadoras en los lugares de trabajo y perpetúan la desigualdad.

No pueden continuar en el tiempo unos diseños organizativos que se desarrollan solamente sobre el modelo hombre, los centros de trabajo deben ser neutrales y en las relaciones laborales se deben de tener en cuenta que los ciclos de vida son distintos para mujeres y hombres y que dichos colectivos no asumen las responsabilidades familiares de la misma forma.

Concluimos, somos del criterio, como hemos expuesto anteriormente, que tener un trabajo remunerado es muy positivo para el desarrollo y la independencia financiera de la persona, por lo tanto no puede continuar en el tiempo la discriminación del sexo femenino en el ámbito laboral, debiendo tener las mujeres acceso a un empleo de calidad en las mismas condiciones que sus compañeros del sexo masculino y en un entorno mixto que contemple las circunstancias particulares de los dos sexos, no solamente la del masculino como ocurre actualmente.

En caso contrario, las mujeres seguirán teniendo un lugar secundario en los centros productivos y se perpetuarán en el tiempo las desigualdades de género al no facilitarles el poder realizar sus proyectos de vida, así como sus aspiraciones profesionales.

\section{Bibliografía}

Álvarez, S. (2015). La autonomía personal y la autonomía relacional. Análisis Filosófico, XXXV (1), 13-26. Recuperado de: https://www.redalyc.org/articulo.oa?id $=340042261002$

Alonso, L. E. (2007). La crisis de la ciudadanía laboral, Barcelona, España: Anthropos Editorial.

Auvergnon, P. (2012). El fenómeno de los trabajadores pobres: revelador de las funciones y tendencias del Derecho Social, Revista Latinoamericana de Derecho Social, 14, 43-78.

Ayala Cañón, L., Cantó Sánchez, O., Martínez López, R., Navarro Ruíz, C. y Romaguera de la Cruz, M. (junio 2020). Informe 6.4. Análisis de las necesidades sociales de mujeres y hombres. Observatorio Social de La Caixa, Recuperado de: https://observatoriosociallacaixa.org/documents/22890/277830/INF 6 CAST 4 MUJERESHOMBRES JUnio.pdf/b7gf6f54-5952-2fo3-b105-0c4474653495

Ayuso, M., \& Chuliá, E. (2018). ¿Hacia la progresiva reducción de la brecha de género en las pensiones contributivas? Documentos de trabajo Instituto BBVA de Pensiones, 22.

Recuperado de:

https://www.jubilaciondefuturo.es/recursos/doc/pensiones/2016060g/esfe/201 8-pensiones-y-brecha-de-genero.pdf 
Beauvoir (de), S. (2019). El segundo sexo. 14. ${ }^{a}$ edición, Madrid, España: Ediciones Cátedra.

Blázquez Rodríguez, M. I. y Montes Muñoz, M. J. (2011). Aspectos actuales en los cuidados de enfermería a la salud de las mujeres. La perspectiva de género en la salud, Madrid, España: Editorial Universitaria Ramón Areces.

Camps, V. (1998). El siglo de las mujeres, Madrid, España: Ediciones Cátedra.

Carrasco Bengoa, C. (2008). Mujeres y trabajo: entre la invisibilidad y la precariedad. En J. Birulés Bertrán, y M. A. Vivas Larruy (Dirs.). Mujer y trabajo: entre la precariedad y la desigualdad. Madrid, España: Consejo General del Poder Judicial.

Carrasquer, P. y Torns, T. (2007). Cultura de la precariedad: conceptualización, pautas y dimensiones. Una aproximación desde la perspectiva de género. Sociedad y utopía. Revista de Ciencias Sociales, (29), 139-156.

Castells, M. (2007). "Capítulo 1. Ser mujer". En M. Castells y M. Subirats, Mujeres y hombres: ¿Un amor imposible?, Madrid, España: Alianza Editorial.

Castillo, J. J. y Agulló, I. (2012). Trabajo y vida en la sociedad de la información, Madrid, España: Los libros de la catarata.

Cebrián López, I. y Moreno Raymundo, G. (2008). La situación de las mujeres en el mercado de trabajo español. Desajustes y retos. Economía Industrial, (367), 121137.

Conde-Pumpido Tourón, M. T. (2007). "La maternidad y la conciliación familiar, dentro de la tutela de la igualdad". En M.T. Conde-Pumpido Tourón (Dir.). Trabajo y familia en la jurisdicción social. Conciliación de la vida familiar y laboral y protección contra la violencia de género, Madrid, España: Consejo General del Poder Judicial.

Federación de Asociaciones de Madres Solteras (S.F.). Las familias monoparentales en España, Madrid, España: Instituto de las Mujeres.

Fernández, E. (2003). Igualdad y Derechos Humanos, Madrid, España: Editorial Tecnos.

Fernández Collados, M. B. (2020). El papel de la negociación colectiva ante el reto de la industria 4.0. En D.T. Kahale Carrillo (Dir.) El impacto de la industria 4.0 en el trabajo: una visión interdisciplinar. Navarra, España: Editorial Aranzadi.

Fundación Felipe González y Fundación Cotec para la innovación. Atlas de oportunidades. Recuperado de: https://www.cotec.es/fundacionfelipegonzalez/oportunidades/proyecto/ 
Hirigoyen, M.-F. (2008). Las nuevas soledades. El reto de las relaciones personales en el mundo de hoy, Barcelona, España: Ediciones Paidós Ibérica.

INE. (2020). Encuesta Continua de Hogares. Recuperado de https://www.ine.es/prensa/ech_2020.pdf

INE. (2019). Encuesta de Condiciones de Vida. Recuperado de https://www.ine.es/prensa/ecv_2019.pdf

ISDI (2016). Digitalización de los profesionales españoles. Informe ISDI. Recuperado de: https://www.isdi.education/sites/default/files/estudio digitalizacion_profesion ales.pdf

Jiménez Sureda, M. (2009). La mujer en la esfera laboral a lo largo de la historia. Manuscrits: Revista d'història moderna. (27) 21-49.

Kierkegaard, S. (1972). El concepto de la angustia. Madrid, España: Espasa Calpe.

Luceño Moreno, L.; Martín García, J.; Rubio Valdehita, S. y Jaén Díaz, M. (2008). Psicología y riesgos laborales emergentes, los riesgos psicosociales. EduPsykhé: Revista de psicología y psicopedagogía, 7(2).

Marías, J. (1979). La justicia social y otras justicias. Madrid, España: Espasa Calpe.

Marina, J. A. (2006). Anatomía del miedo: un tratado sobre la valentía. Barcelona, España: Anagrama.

Martín Gaite, C. (2017). Usos amorosos de la postguerra española. Decimoctava edición. Barcelona, España: Anagrama.

Martínez, M.C. y Paterna, C. (2009). Perspectiva de género aplicada a la conciliación. En M.C. Martínez Martínez (Coord.). Género y conciliación de la vida familiar y laboral, Murcia, España: Universidad de Murcia.

Medina-Vicent, M. (2014). El papel de las trabajadoras durante la industrialización europea del Siglo XIX. Construcciones discursivas del movimiento obrero en torno al sujeto "mujeres". Fòrum de Recerca, (19), 149-163.

Millán Vázquez de la Torre, M. G.; Santos Pita, M. P. y Pérez Naranjo, L. M. (2015). Análisis del mercado laboral femenino en España: evolución y factores socioeconómicos determinantes del empleo. Papeles de población, 21(84).

Millett, K. (2010). Política sexual, Madrid, España: Ediciones Cátedra.

Molino (Del), S. (2018). La España vacía. Viaje por un país que nunca fue. Decimotercera reimpresión. Madrid, España: Turner Publicaciones.

Oficina Nacional de Prospectiva y Estrategia del Gobierno de España (Coord.) (2021). España 2050: Fundamentos y propuestas para un Estrategia Nacional de Largo Plazo. Madrid, España: Ministerio de la Presidencia. Recuperado de: 
https://www.lamoncloa.gob.es/presidente/actividades/Documents/2021/200521Estrategia Espana 2050.pdf

Oñate, P. (2010). El sistema político. En M. García Ferrando (Coord.). Pensar nuestra sociedad globalizada. Una invitación a la sociología, Valencia, España: Tirant lo Blanch.

Ovejero Bernal, A. (2006). Psicología del trabajo en un mundo globalizado. Cómo hacer frente al mobbing y al estrés laboral. Madrid, España: Biblioteca Nueva.

Pazos Morán, M. (1 de septiembre de 2009). Las mujeres en el cambio de modelo productivo. El País. Recuperado de https://elpais.com/diario/200g/og/o1/opinion/1251756004_850215.html

Rojas Marcos, L. (2010). Superar la adversidad. Madrid, España: Espasa.

Rubio, A. y Gil, J. M. (2012). Dignidad e igualdad de derechos. El acoso en el trabajo. Madrid, España: Dykinson.

Ruíz, P. M. (2020). Industria 4.0, de la tecnología a los retos para la educación superior. En D.T. Kahale Carrillo (Dir.) El impacto de la industria 4.o en el trabajo: una visión interdisciplinar, Navarra, España: Aranzadi.

San Martín Mazzucconi, C. (2020). La conciliación de la vida laboral y extralaboral en la IV revolución industrial. En D.T. Kahale Carrillo (Dir.) El impacto de la industria 4.o en el trabajo: una visión interdisciplinar, Navarra, España: Aranzadi.

Sarasúa, C. y Gálvez, L. (2003). Mujeres y hombres en los mercados de trabajo. ¿Privilegios o eficiencia? En C. Sarasúa, y L. Gálvez, (Eds.). ¿Privilegios o eficiencia? Mujeres y hombres en los mercados de trabajo. San Vicente del Raspeig, España: Publicaciones de la Universidad de Alicante.

Valiente Fernández, C. (1998). La liberación del régimen franquista: La Ley de 22 de julio de 1961 sobre derechos políticos profesionales y de trabajo de la mujer. Historia social. (31), 45-65.

Viguer Seguí, P. y Serra Desfilis, E. (1996). Nivel socioeconómico y calidad del entorno familiar en la infancia. Anales de Psicología, 12(2)197-205.

Vilar Rodríguez, M. (2012). ¿De aquellas arenas estos lodos?: el mercado de trabajo en España desde una perspectiva histórica. Revista galega de economía: Publicación Interdisciplinar da Facultade de Ciencias Económicas e Empresariais 21(1), 115146. 
Cómo referenciar este artículo/How to reference this article:

Uris Lloret, J.M. (2022). La autonomía de la mujer relación con el trabajo remunerado. iQUAL. Revista de Género e lgualdad, 5, 145-167, doi: 10.6018/iqual.489651

Uris Lloret, J.M. (2022). La autonomía de la mujer relación con el trabajo remunerado. [Woman's autonomy in relation to paid work] iQUAL. Revista de Género e lgualdad, 5, 145-167, doi: 10.6018/iqual.489651 